\title{
Lithium Detection in a Binary Al-20 wt\% Li Alloy Powder with a New Windowless SDD EDS Detector and EELS
}

\author{
Vladimir P. Oleshko ${ }^{1}$, Stephen M. Seddio ${ }^{2}$, Robert H. Newton², Christopher L. Soles ${ }^{2}$, and Jabez J. \\ McClelland ${ }^{2}$ \\ 1. National Institute of Standards and Technology, Gaithersburg, MD, USA \\ 2. Thermo Fisher Scientific, Madison, WI, USA
}

The introduction of silicon drift (SDD) EDS detectors has enabled the design and production of X-ray detectors that need only be cooled to $-25^{\circ} \mathrm{C}$ and could therefore be cooled by Peltier devices instead of liquid nitrogen. This reduction in cooling overhead enabled the practical production of windowless X-ray detectors for use in the SEM, drastically boosting light element analysis with ultra-soft X-rays below $1 \mathrm{keV}$. Li is the lightest element that can generate a characteristic X-ray by electron interaction, but $>99 \%$ of Li X-rays are expected to be absorbed in a 0.3 $\mu \mathrm{m}$ thick polymer window [3]. Windowless EDS detectors designed to collect ultra-soft X-rays $(<200 \mathrm{eV})$ eliminate the absorption of $\mathrm{Li} \mathrm{K \alpha}$ (and other soft) X-rays in the window.

Although lithium detection in metallic Li by SDD EDS was demonstrated a few years ago, detection challenges remain [4]. Metallic Li and many lithium compounds are highly reactive and, upon being exposed to air, readily react with it. The escape depth of $\mathrm{Li}$ is less than $20 \mathrm{~nm}$ and, as the surface accumulates the oxidation products, the Li K $\alpha$ X-rays are increasingly absorbed in the oxidized layer. Samples must be prepared and maintained in a manner that allows Li X-rays to escape the sample. Additionally, the transition from the Li Ll to K shells is not allowed by the selection rule. Therefore unless bonding interaction causes the L1 electron to take on more L2,3 characteristics [2], Li X-ray generation cannot occur.

In this work, we report on lithium detection with a new windowless UltraDry SDD EDS detector (Thermo Fisher Scientific) using a commercial Al-20 wt \% Li alloy powder as a reference material [5]. Prior to EDS analyses, the microstructure and local chemical compositions of the powders were characterized by conventional TEM, HRTEM, and STEM-EELS. It was found that the alloy powders consist of agglomerated 1-4 $\mu \mathrm{m}$ particles (Figs. $1 \mathrm{a}$ and $1 \mathrm{~b}$ ) composed of a partially disordered $\beta$-AlLi phase crystallized in NaT1 type structure (space group $\mathrm{Fd} 3 \mathrm{~m})$, which has a homogeneity range between about 45 and 56 at\% $\mathrm{Li}[6] . \mathrm{Li} \mathrm{K \alpha}(55 \mathrm{eV})$ and $\mathrm{Al} \mathrm{L}_{2,3}(72 \mathrm{eV})$ are only separated by $17 \mathrm{eV}$. In the EDS spectra acquired from Li and Al metals, the Li Ka peak is centered at 59 $\mathrm{eV}$, and $\mathrm{Al} \mathrm{L}_{2,3}$ is centered at $75 \mathrm{eV}$. In the EDS spectra acquired from the $\mathrm{Al}-20 \mathrm{wt} \% \mathrm{Li}$ alloy, a peak is centered at $\sim 70 \mathrm{eV}$. We interpret this peak to be an unresolved $\mathrm{Li} \mathrm{K \alpha}+\mathrm{Al}_{2,3}$ peak. This is consistent with EELS spectra collected on the same alloy powders with an estimated atomic $\mathrm{Li} / \mathrm{Al}$ ratio of about 0.74 . Fig. $2 \mathrm{a}$ shows for comparison also an X-ray spectrum a metallic Li foil, which could react with gas molecules in the SEM chamber to yield the non-Li peaks above $200 \mathrm{eV}$. This work emphasizes the usefulness of cross-instrumentational comparison of analyses made by ultra-soft EDS and S/TEM-EELS on the same reference materials for reliable interpretation of the results and assessing the detector performance and the possibility to separate close X-ray peaks such as the $\mathrm{Li} \mathrm{K} \alpha$ and the $\mathrm{Al} \mathrm{L}_{2,3}$ lines.

References:

[1] S Burgess et al, Microsc Microanal 19 (S2) (2013), p. 1142-43; ibid, 22 (S3) (2016) 112-13.

[2] S Burgess et al, Microscopy Today 25 (2017), p. 20-28.

[3] B Henke et al, Atom Data Nucl Data 54 (1993), p. 181-342.

[4] P Hovington et al, Scanning 38 (2016), p. 571-78.

[5] Certain equipment, instruments or materials are identified in this paper in order to adequately specify the experimental details. Such identification does not imply any endorsement by the NIST nor does it imply the materials are the best available for the purpose. 
[6] K Kishio, JO, Brittain, J Phys Chem Solids 40 (1979) 93W40.

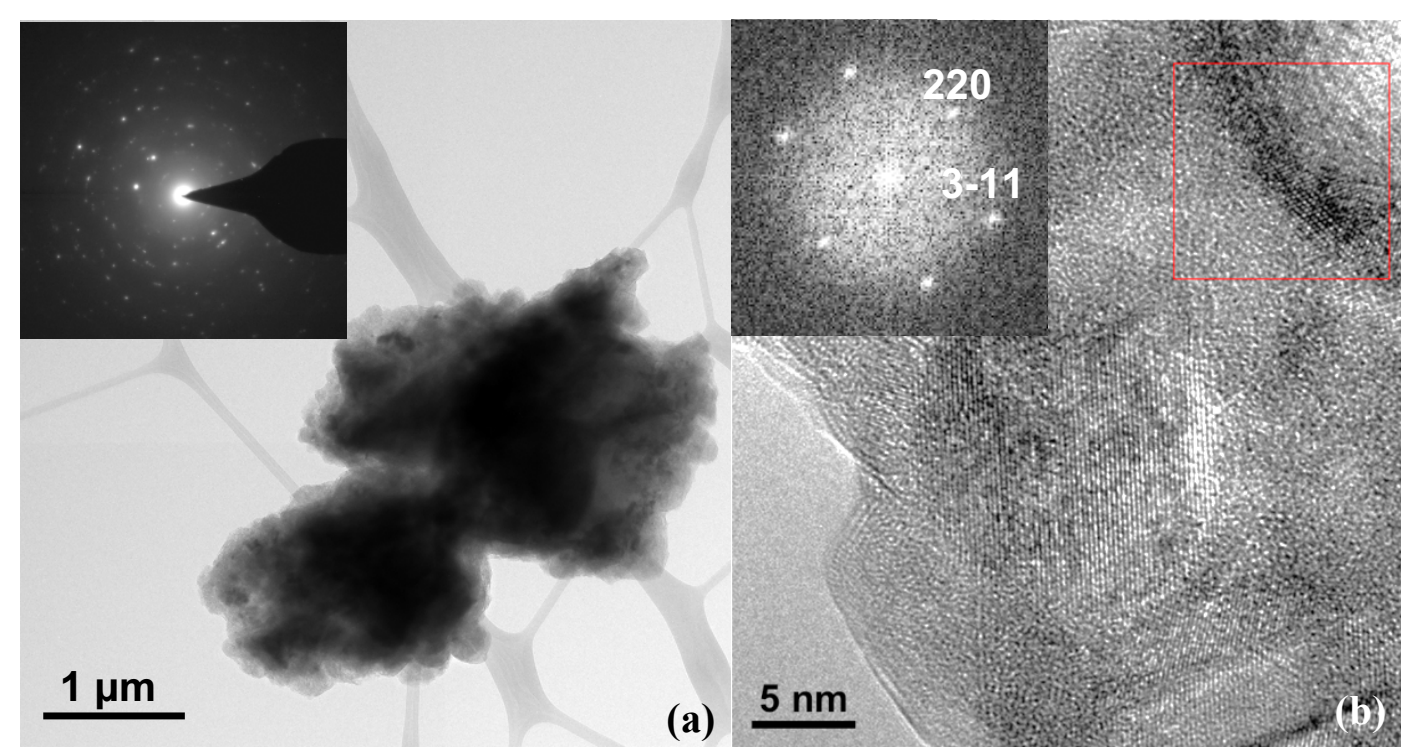

Figure 1. Al-20 wt\% Li alloy powder. (a) BF-TEM, 2-3 $\mu \mathrm{m}$ agglomerated alloy particles. SAED pattern (inset) shows discrete point reflections from randomly oriented crystallites assigned to $\beta$-AlLi phase. (b) HRTEM, near the edge of a powder aggregate reveals lattice fringes from $\beta$-AlLi nanocrystallites embedded into the partially amorphous matrix. FFT from the region marked by red squire (inset) indicates $\beta$-AlLi (220) and (311) point reflections, the zone axis $\mathrm{B}=[-114]$.

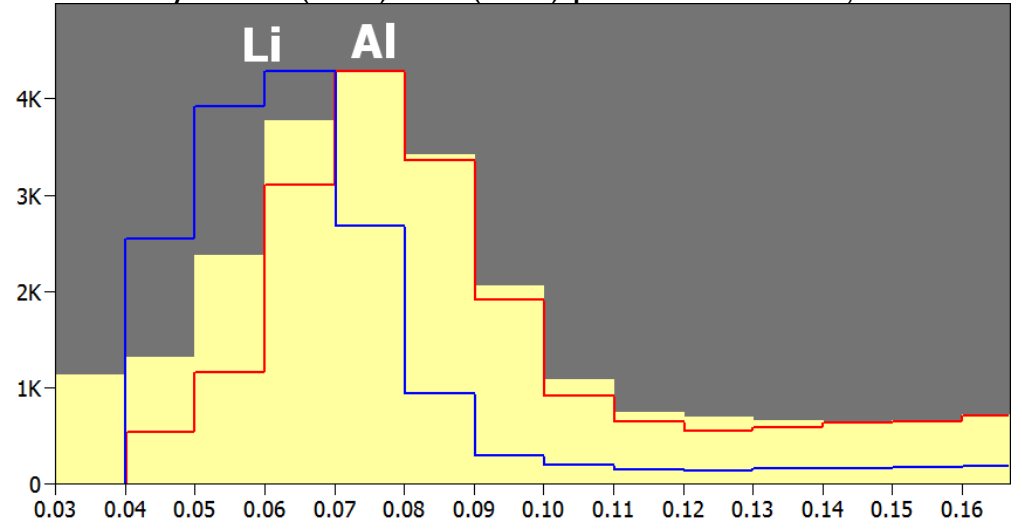

(a)

(b)

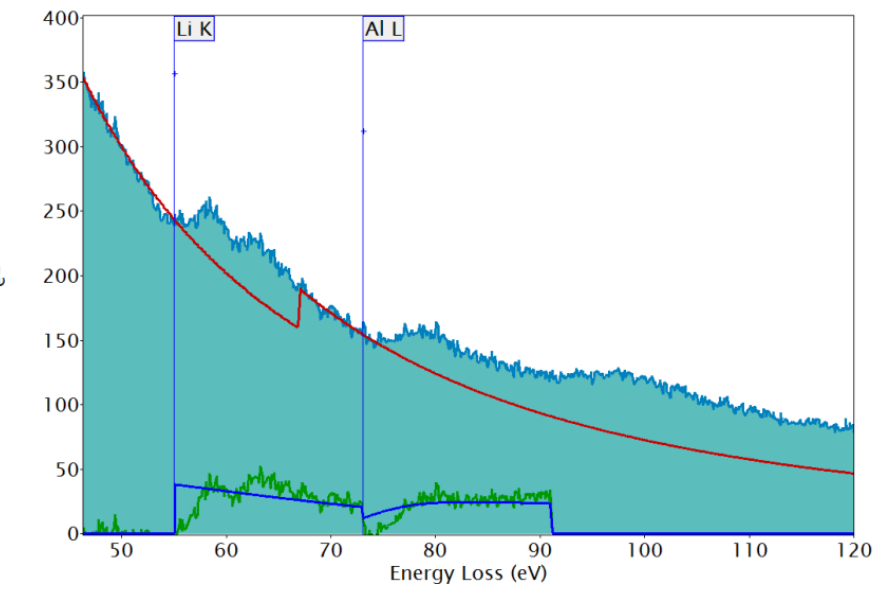

Figure 2. (a) X-ray spectra of the Al-20 wt\% Li alloy powder (yellow), metallic $\mathrm{Al}$ (red) and Li metal (blue). (b) EEL spectrum of the same powder showing the Li K-edge at 55 $\mathrm{eV}$ and the $\mathrm{Al} \mathrm{L}_{2,3}$ edge at $72 \mathrm{eV}$ energy loss, respectively. Fitted power law backgrounds for both edges are indicated by red line. Green curves represent the net edges separated from the extrapolated backgrounds. Black curves show the calculated Hartree-Slater scattering cross-sections for Li and Al integrated over the same width energy windows. 